\title{
Blowup of solutions of dissipative nonlinear wave equations
}

(Dedicated to Professor Rentaro Agemi on his 60th birthday)

\section{Masahito OHTA*}

(Received December 6, 1995)

\begin{abstract}
We consider the blowup problem for some dissipative nonlinear wave equations. Under appropriate conditions, it is shown that if the initial data belongs to the so called unstable set, then the solution blows up in a finite time as well as the nondissipative case. In the proof, we propose a new method instead of the usual concavity method. In particular, we use a blowup result for some ordinary differential inequalities of the second order and the monotonicity of some Rayleigh type quotients.
\end{abstract}

Key words: blowup, dissipative nonlinear wave equations, unstable set.

\section{Introduction and Main Results}

In this paper, we consider the blowup problem for nonlinear evolution equations of the form:

$$
\begin{aligned}
& u^{\prime \prime}(t)+\delta u^{\prime}(t)+\partial \varphi(u(t))-\partial \psi(u(t))=0, \quad t \geq 0 \\
& u(0)=u_{0}, \quad u^{\prime}(0)=u_{1}
\end{aligned}
$$

in a real Hilbert space $H$. Here, $\partial \varphi$ and $\partial \psi$ are single valued subdifferentials of lower semicontinuous convex functions $\varphi$ and $\psi$ from $H$ to $(-\infty,+\infty]$ with $\varphi, \psi \not \equiv+\infty, u^{\prime \prime}(t)$ and $u^{\prime}(t)$ stand for $d^{2} u(t) / d t^{2}$ and $d u(t) / d t$, respectively, and $\delta$ is a positive constant.

Equation (1.1) includes as special cases dissipative nonlinear wave equations:

$$
\begin{gathered}
u_{t t}+\delta u_{t}-\left(\alpha+\beta\|\nabla u\|_{L^{2}(\Omega)}^{2}\right) \Delta u-\mu|u|^{q-2} u=0, \\
t \geq 0, \quad x \in \Omega, \\
u(t, x)=0, \quad t \geq 0, \quad x \in \partial \Omega,
\end{gathered}
$$

1991 Mathematics Subject Classification : 35L70, 35B05.

*Partially supported by JSPS Research Fellowships for Young Scientists. 
where $\alpha \geq 0, \beta \geq 0, \alpha^{2}+\beta^{2}>0, \mu>0, q>2$ and $\Omega$ is a domain in $\mathbf{R}^{n}$.

In the case of $\delta=0$, the blowup problem for (1.1) has been studied by many authors (see, e.g., [16], [5], [11], [14], [1], [9], [2] and [7]). In particular, under appropriate conditions on $\varphi$ and $\psi$, it is shown that if the initial data $\left(u_{0}, u_{1}\right)$ belongs to the so called unstable set $V$ defined by (1.8) below, then the solution of (1.1) $(1.2)$ with $\delta=0$ blows up in a finite time (see, e.g., [14] and [9], [2] and [7]). However, when $\delta>0$, there are not so many works dealing with the blowup problem for (1.1). Recently, Ikehata [6] and Ikehata and Suzuki [8] studied the blowup problem for (1.3)-(1.4), and under certain conditions on $\alpha, \beta$ and $q$ they showed that if $\delta>0$ is small and the initial data belongs to some subset $V_{\delta}$ of the unstable set $V$, then the solution blows up in a finite time. Here, we note that $V_{\delta}$ is not equal to $V$ if $\delta>0$.

Our main purpose in this paper is to remove the above technical restrictions on $\delta$ and initial data imposed in [6] and [8]. That is, under appropriate conditions on $\varphi$ and $\psi$, for any $\delta>0$, we shall show that if the initial data $\left(u_{0}, u_{1}\right)$ belongs to the unstable set $V$, then the solution of (1.1) (1.2) blows up in a finite time as well as the case of $\delta=0$. Here, we note that this problem is related to the instability of some stationary solutions of (1.1) (see [14] and [2]).

To state our main result, we need to prepare some notations. We denote by $|\cdot|_{H}$ and $(\cdot, \cdot)_{H}$ the norm and the inner product of $H$, respectively. Without loss of generality, we can assume that $\varphi(0)=0$ and $\psi(0)=0$. For the definition and basic properties of subdifferentials, see, e.g., Section 2 of Ishii [9]. For $u \in D(\partial \varphi) \cap D(\partial \psi)$ and $v \in H$, we put

$$
\begin{aligned}
& J(u)=\varphi(u)-\psi(u), \\
& K(u)=(u, \partial \varphi(u))_{H}-(u, \partial \psi(u))_{H}, \\
& E(u, v)=(1 / 2)|v|_{H}^{2}+J(u),
\end{aligned}
$$

and we define the unstable set $V$ as follows:

$$
V=\{(u, v) \in[D(\partial \varphi) \cap D(\partial \psi)] \times H ; E(u, v)<d, K(u)<0\},
$$

where

$$
d=\inf \{J(u) ; u \in D(\partial \varphi) \cap D(\partial \psi), K(u)=0, u \neq 0\} .
$$


In what follows, we assume that

(A1) there exists $q>2$ such that $q J(u)-K(u) \geq 0$ for all $u \in D(\partial \varphi) \cap$ $D(\partial \psi)$.

We put $Q(u)=q J(u)-K(u)$. From (1.9) and (A1), we have

$$
d=\inf \{(1 / q) Q(u) ; u \in D(\partial \varphi) \cap D(\partial \psi), K(u)=0, u \neq 0\} \geq 0 .
$$

Furthermore, we also assume that the following (A2) holds.

(A2) $d=\inf \{(1 / q) Q(u) ; u \in D(\partial \varphi) \cap D(\partial \psi), K(u) \leq 0, u \neq 0\}$.

Remark 1.1. When $\varphi$ and $\psi$ are homogeneous functions of degree $p$ and $q>1$ respectively, we have $K(u)=p \varphi(u)-q \psi(u)$ and $q J(u)-K(u)=$ $(q-p) \varphi(u)$. Thus, (A1) is satisfied if $p \leq q, q>2$ and $\varphi(u) \geq 0$ for $u \in H$. In addition, if $\varphi(u)>0$ for $u \neq 0$, then (A2) is satisfied (see Proof of Theorem 1.2 in Section 2). We also note that $d=0$ if $p=q$.

Remark 1.2. By (A1), we have $(1 / q) K(u) \leq J(u) \leq E(u, v)$, so in the case when $d=0$, we have $V=\{(u, v) \in[D(\partial \varphi) \cap D(\partial \psi)] \times H ; E(u, v)<0\}$.

In this paper, we consider the following class of solutions of (1.1) (1.2).

Definition A function $u(t) \in C^{1}([0, T] ; H)$ is said to be a strong solution of (1.1)-(1.2) in $[0, T]$ if $u^{\prime \prime}(t), \partial \varphi(u(t)), \partial \psi(u(t)) \in L^{2}(0, T ; H), K(u(t)) \in$ $C([0, T] ; \mathbf{R})$, and $u(t)$ satisfies $(1.1)-(1.2)$.

Remark 1.3. When $\varphi$ and $\psi$ are homogeneous functions of degree $p$ and $q>1$ respectively, we have $K(u)=p \varphi(u)-q \psi(u)$. Thus, the continuity of $K(u(t))$ follows from $u(t) \in C^{1}([0, T] ; H)$ and $\partial \varphi(u(t)), \partial \psi(u(t)) \in$ $L^{2}(0, T ; H)$.

In what follows, we assume that the problem (1.1)-(1.2) with $\left(u_{0}, u_{1}\right) \in$ $V$ has a strong solution in $[0, T]$ for some $T>0$. We now state our main result in this paper.

Theorem 1.1 Let $\delta>0$. Assume that (A1) and (A2) are satisfied. Then any strong solution of $(1.1)-(1.2)$ with $\left(u_{0}, u_{1}\right) \in V$ can not be continued to $[0,+\infty)$ as a strong solution of (1.1)-(1.2).

Combining Theorem 1.1 and Remark 1.1, we obtain the following result. Theorem 1.2 Let $\delta>0$ and let $\varphi$ and $\psi$ be homogeneous functions of degree $p$ and $q$, respectively. Suppose that $1<p \leq q, q>2$, and $\varphi(u)>0$ 
for $u \in H \backslash\{0\}$. Then any strong solution of (1.1)-(1.2) with $\left(u_{0}, u_{1}\right) \in V$ can not be continued to $[0,+\infty)$ as a strong solution of (1.1)-(1.2).

Most of the proofs for the case of $\delta=0$ are based on the so called concavity method, which is useful for the case of $\delta=0$ (see, e.g., [11], [14] and [9]). However, it seems that this method does not give sharp results for the case of $\delta>0$. So, in the proof of Theorem 1.1, we use a blowup result for some ordinary differential inequalities of the second order by $\mathrm{Li}$ and Zhou [12] and Souplet [15] (see Lemma 2.3 in Section 2), instead of the concavity method. Moreover, the monotonicity of some Rayleigh type quotients plays an important role in the proof of Theorem 1.1 (see Lemma 2.4 in Section 2). A similar technique was previously used by Fujii and the author [4] for parabolic equations. Our method works for all $\delta>0$ and $\left(u_{0}, u_{1}\right) \in V$.

The plan in this paper is as follows. In Section 2 we prove Theorems 1.1 and 1.2 together with several lemmas. In Section 3 we apply Theorems 1.1 and 1.2 to the blowup problem for dissipative nonlinear wave equations (1.3)-(1.4).

\section{Proof of Theorems}

In this section, we give the proof of Theorems 1.1 and 1.2. First, we prove Theorem 1.1. The proof of Theorem 1.1 consists of two steps. The first step is to show the following lemma, which is almost the same as that in [14], [9], [6] and [8]. Our new idea is introduced in the second step.

Lemma 2.1 Assume (A1) and (A2). Let $u(t)$ be a strong solution of (1.1)-(1.2) with $\left(u_{0}, u_{1}\right) \in V$ in $[0, T]$. If we put $I(t)=(1 / 2)|u(t)|_{H}^{2}$ and $E(t)=E\left(u(t), u^{\prime}(t)\right)$, then we have $I^{\prime \prime}(t)+\delta I^{\prime}(t) \geq(1+q / 2)\left|u^{\prime}(t)\right|_{H}^{2}$ $+q(d-E(t))$ for all $t \in[0, T]$.

To prove Lemma 2.1, we need one lemma, which is also used by many authors.

Lemma 2.2 Assume (A1) and (A2). Let $u(t)$ be a strong solution of (1.1)-(1.2) with $\left(u_{0}, u_{1}\right) \in V$ in $[0, T]$. Then, $E\left(u(t), u^{\prime}(t)\right)<d$ and $K(u(t))<0$ for all $t \in[0, T]$. 
Proof. We put $E(t)=E\left(u(t), u^{\prime}(t)\right)$. Multiplying (1.1) by $u^{\prime}(t)$, we have

$$
E^{\prime}(t)=-\delta\left|u^{\prime}(t)\right|_{H}^{2}
$$

for almost all $t \in[0, T]$. So, we have $E(t) \leq E(0)<d$ for all $t \in[0, T]$. If $d=0$, Lemma 2.2 follows from Remark 1.2. Thus, we assume that $d>0$. To conclude the proof, we have only to show that $K(u(t))<0$ holds for all $t \in[0, T]$. Suppose that there exists $t_{0} \in(0, T]$ such that $K\left(u\left(t_{0}\right)\right)=0$ and $K(u(t))<0$ for all $t<t_{0}$. Then, it follows from (A2) that $Q(u(t)) \geq q d$ for all $t<t_{0}$. By the continuity of $t \mapsto Q(u(t)), Q(0)=0$ (we always assume that $\varphi(0)=0$ and $\psi(0)=0)$ and $d>0$, we have $u\left(t_{0}\right) \neq 0$. Therefore, from $(1.9)$, we have $d \leq J\left(u\left(t_{0}\right)\right) \leq E\left(t_{0}\right)<d$, which is a contradiction. Hence, by the continuity of $t \mapsto K(u(t))$, we have $K(u(t))<0$ for all $t \in[0, T]$. This completes the proof.

Proof of Lemma 2.1 Multiplying (1.1) by $u(t)$, we have

$$
\left(u(t), u^{\prime \prime}(t)\right)_{H}+\delta\left(u(t), u^{\prime}(t)\right)_{H}+K(u(t))=0 .
$$

Using the identities:

$$
I^{\prime}(t)=\left(u(t), u^{\prime}(t)\right)_{H}, \quad I^{\prime \prime}(t)=\left|u^{\prime}(t)\right|_{H}^{2}+\left(u(t), u^{\prime \prime}(t)\right)_{H}
$$

and

$$
K(u)=q E(u, v)-(q / 2)|v|_{H}^{2}-Q(u),
$$

we have

$$
I^{\prime \prime}(t)+\delta I^{\prime}(t)=(1+q / 2)\left|u^{\prime}(t)\right|_{H}^{2}-q E(t)+Q(u(t)),
$$

Lemma 2.1 follows from (2.5), (A2) and Lemma 2.2.

The rest of the proof of Theorem 1.1 seems new. The following two lemmas play important roles in this part.

Lemma 2.3 (Li and Zhou [12] and Souplet [15]) Let $\delta>0$. Suppose that $h(t)$ satisfies

$$
\begin{aligned}
& h^{\prime \prime}(t)+\delta h^{\prime}(t) \geq C_{0} h^{1+\alpha}(t), \quad t>0, \\
& h(0)>0, \quad h^{\prime}(0)>0
\end{aligned}
$$

for some positive constants $C_{0}$ and $\alpha$. Then, $h(t)$ can not exist for all $t>0$. 
Lemma 2.3 is a special case of Theorem 3.1 in Li and Zhou [12] and Theorem 1.1 in Souplet [15] (see also Zhou [17] and Mizoguchi, Ninomiya and Yanagida [13]). Our main task in this paper is to show the following lemma.

Lemma 2.4 Assume (A1) and (A2). Suppose that a strong solution $u(t)$ of (1.1)-(1.2) with $\left(u_{0}, u_{1}\right) \in V$ exists globally in time. Then, there exists $t_{1}>0$ such that $I(t)>0, I^{\prime}(t)>0$ and

$$
\frac{d}{d t} \frac{E\left(u(t), u^{\prime}(t)\right)-d}{I^{\gamma}(t)} \leq 0
$$

for almost all $t \in\left[t_{1},+\infty\right)$, where $\gamma=(q+2) / 4$ and $I(t)=(1 / 2)|u(t)|_{H}^{2}$.

Proof. We put

$$
E(t)=E\left(u(t), u^{\prime}(t)\right), \quad F(t)=\delta I^{\prime}(t)+(1+q / 2)(E(t)-d) .
$$

Then, from Lemma 2.1 and (2.1), we have

$$
\begin{aligned}
F^{\prime}(t) & =\delta I^{\prime \prime}(t)+(1+q / 2) E^{\prime}(t) \geq-\delta^{2} I^{\prime}(t)+\delta q(d-E(t)) \\
& =-\delta F(t)+\delta(q / 2-1)(d-E(t)),
\end{aligned}
$$

from which it follows that

$$
\begin{aligned}
\left(e^{\delta t} F(t)\right)^{\prime} & =e^{\delta t}\left(F^{\prime}(t)+\delta F(t)\right) \geq \delta(q / 2-1)(d-E(t)) e^{\delta t} \\
& \geq \delta(q / 2-1)(d-E(0)) e^{\delta t}
\end{aligned}
$$

Integrating $(2.10)$ yields

$$
\begin{gathered}
F(t) \geq e^{-\delta t}\{F(0)-(q / 2-1)(d-E(0))\} \\
+(q / 2-1)(d-E(0)) .
\end{gathered}
$$

Since $E(0)<d$ and $q>2$, there exists $t_{1}>0$ such that

$$
F(t)>0, \quad t \geq t_{1} / 2 .
$$

In particular, from (2.12), we have

$$
\begin{aligned}
\delta I^{\prime}(t) & \geq(1+q / 2)(d-E(t)) \\
& \geq(1+q / 2)(d-E(0))>0, \quad t \geq t_{1} / 2,
\end{aligned}
$$

from which it follows that

$$
I(t)>0 \quad \text { and } \quad I^{\prime}(t)>0, \quad t \geq t_{1} .
$$


For $t \geq t_{1}$, we have

$$
\frac{d}{d t} \frac{E(t)-d}{I^{\gamma}(t)}=I^{-\gamma-1}(t)\left\{E^{\prime}(t) I(t)-\gamma I^{\prime}(t)(E(t)-d)\right\}
$$

and from (2.1), (2.12) and (2.14), we have for $\gamma=(q+2) / 4$

$$
\begin{aligned}
& E^{\prime}(t) I(t)-\gamma I^{\prime}(t)(E(t)-d) \\
& \quad \leq-(\delta / 2)|u(t)|_{H}^{2}\left|u^{\prime}(t)\right|_{H}^{2}+(\delta / 2)\left(I^{\prime}(t)\right)^{2} \leq 0 .
\end{aligned}
$$

Here, we have used the fact $I^{\prime}(t)=\left(u(t), u^{\prime}(t)\right)_{H}$ and the Cauchy-Schwarz inequality. Lemma 2.4 follows from (2.14)-(2.16).

We are now in a position to complete the proof of Theorem 1.1.

Proof of Theorem 1.1 Suppose that a strong solution $u(t)$ of $(1.1)-(1.2)$ with $\left(u_{0}, u_{1}\right) \in V$ exists globally in time. Then, from Lemma 2.4, there exists $t_{1}>0$ such that

$$
I(t)>0, \quad I^{\prime}(t)>0, \quad d-E(t) \geq C_{1} I^{\gamma}(t), \quad t \geq t_{1},
$$

where we put $C_{1}=\left(d-E\left(t_{1}\right)\right) / I^{\gamma}\left(t_{1}\right)$. Here, from Lemma 2.1, we have

$$
I^{\prime \prime}(t)+\delta I^{\prime}(t) \geq q(d-E(t)) \geq q C_{1} I^{\gamma}(t), \quad t \geq t_{1} .
$$

Since $\gamma=(q+2) / 4>1, C_{1}>0, I\left(t_{1}\right)>0$ and $I^{\prime}\left(t_{1}\right)>0$, it follows from Lemma 2.3 that $I(t)$ can not exist for all $t>0$. However, this contradicts the assumption that the strong solution $u(t)$ exists globally in time. Hence, we obtain Theorem 1.1.

Finally, we prove Theorem 1.2.

Proof of Theorem 1.2 As stated in Remark 1.1, when $\varphi$ and $\psi$ are homogeneous functions of degree $p$ and $q>1$ respectively, we have $K(u)=$ $p \varphi(u)-q \psi(u)$ and $Q(u)=q J(u)-K(u)=(q-p) \varphi(u)$, so we have only to check (A2). To do so, it suffices to show that $K(u)<0$ implies $q d \leq Q(u)=(q-p) \varphi(u)$. If $p=q$, we have $d=0$, so (A2) holds. Thus, we assume that $p<q$. Then, it follows from $K(u)<0$ that $u \neq 0, \varphi(u)>0$ and $\psi(u)>0$. Since $K(\lambda u)=p \lambda^{p} \varphi(u)-q \lambda^{q} \psi(u)$ and $K(u)<0$, there exists $\lambda_{1} \in(0,1)$ such that $K\left(\lambda_{1} u\right)=0$, so it follows from (1.10) that $q d \leq Q\left(\lambda_{1} u\right)=(q-p) \varphi\left(\lambda_{1} u\right)=(q-p) \lambda_{1}^{p} \varphi(u) \leq(q-p) \varphi(u)$. This completes the proof. 


\section{Examples}

In this section, we apply Theorems 1.1 and 1.2 to the blowup problem for dissipative nonlinear wave equations (1.3)-(1.4).

Let $\Omega$ be a bounded domain in $\mathbf{R}^{n}$ with smooth boundary $\partial \Omega$ and let $H=L^{2}(\Omega)$. For $\alpha \geq 0, \beta \geq 0, \alpha^{2}+\beta^{2}>0, \mu>0$ and $q>2$, we define functions $\varphi$ and $\psi$ from $H$ to $(-\infty,+\infty]$ by

$$
\varphi(u)= \begin{cases}(\alpha / 2)\|\nabla u\|_{L^{2}(\Omega)}^{2}+(\beta / 4)\|\nabla u\|_{L^{2}(\Omega)}^{4}, & \text { if } u \in H_{0}^{1}(\Omega), \\ +\infty, & \text { otherwise }\end{cases}
$$

and

$$
\psi(u)= \begin{cases}(\mu / q)\|u\|_{L^{q}(\Omega)}^{q}, & \text { if } u \in L^{q}(\Omega), \\ +\infty, & \text { otherwise }\end{cases}
$$

respectively. It is easy to see that the functions $\varphi$ and $\psi$ are lower semicontinuous convex and $\not \equiv+\infty$, and that $\partial \varphi$ and $\partial \psi$ are single valued and given by

$$
\partial \varphi(u)=-\left(\alpha+\beta\|\nabla u\|_{L^{2}(\Omega)}^{2}\right) \Delta u \quad \text { and } \quad \partial \psi(u)=\mu|u|^{q-2} u .
$$

Then, (1.3)-(1.4) is reduced to (1.1). The local existence of strong solutions for (1.3)-(1.4) is already established (see, e.g., [3] and [7]). Let $\alpha>0$, $\beta \geq 0, q>2$ and $q \leq 1+n /(n-2)$ if $n \geq 3$. Then, for any $\left(u_{0}, u_{1}\right) \in$ $\left[H^{2}(\Omega) \cap H_{0}^{1}(\Omega)\right] \times H_{0}^{1}(\Omega)$, there exists a unique strong solution of (1.3)(1.4) and $[1.2)$ in $[0, T]$ for some $T>0$. Applying Theorems 1.1 and 1.2 and using the notations (1.5) (1.9), we have the following results.

Theorem 3.1 Let $\delta>0, \alpha>0, \beta=0$ and $\mu>0$. Assume $q>2$ and $q \leq 1+n /(n-2)$ if $n \geq 3$. If $\left(u_{0}, u_{1}\right) \in\left[H^{2}(\Omega) \cap H_{0}^{1}(\Omega)\right] \times H_{0}^{1}(\Omega)$ satisfies $E\left(u_{0}, u_{1}\right)<d$ and $K\left(u_{0}\right)<0$, then the strong solution of (1.3)-(1.4) and (1.2) can not be continued to $[0,+\infty)$ as a strong solution of $(1.3)-(1.4)$ and (1.2).

Theorem 3.2 Let $\delta>0, \alpha>0$ and $\beta>0$. Assume $n=3, q=4$ and $\mu>\beta C(\Omega)^{-1}$, where $C(\Omega)=\sup \left\{\|u\|_{L^{4}(\Omega)}^{4} /\|\nabla u\|_{L^{2}(\Omega)}^{4} ; u \in H_{0}^{1}(\Omega), u \neq 0\right\}$. If $\left(u_{0}, u_{1}\right) \in\left[H^{2}(\Omega) \cap H_{0}^{1}(\Omega)\right] \times H_{0}^{1}(\Omega)$ satisfies $E\left(u_{0}, u_{1}\right)<d$ and $K\left(u_{0}\right)<$ 0 , then the strong solution of (1.3)-(1.4) and (1.2) can not be continued to $[0,+\infty)$ as a strong solution of (1.3)-(1.4) and (1.2).

Theorem 3.3 Let $\delta>0, \alpha=0$ and $\beta>0$. Assume $n=3, q=4$ and 
$\mu>\beta C(\Omega)^{-1}$. If $\left(u_{0}, u_{1}\right) \in\left[H^{2}(\Omega) \cap H_{0}^{1}(\Omega)\right] \times H_{0}^{1}(\Omega)$ satisfies $E\left(u_{0}, u_{1}\right)<$ 0 , then the strong solution of (1.3)-(1.4) and (1.2) can not be continued to $[0,+\infty)$ as a strong solution of (1.3)-(1.4) and (1.2).

Theorems 3.1 and 3.3 follow immediately from Theorem 1.2 (see also Remarks 1.1 and 1.2 for Theorem 3.3). Under the conditions in Theorem 3.2, (A1) and (A2) are easily verified similarly to the proof of Theorem 1.2, so Theorem 3.2 follows from Theorem 1.1. Theorems 3.1 and 3.2 improve Theorem 4.2 in Ikehata and Suzuki [8] and Theorem 3.2 in Ikehata [6], respectively. We also note that under the conditions in Theorem 3.3 , for the strong solution $u(t)$ of (1.3)-(1.4) and (1.2), it follows from $J(u(t)) \leq E\left(u(t), u^{\prime}(t)\right) \leq E\left(u_{0}, u_{1}\right)$ that $\beta\|\nabla u(t)\|_{L^{2}(\Omega)}^{4} \leq \mu\|u(t)\|_{L^{4}(\Omega)}^{4}+$ $4 E\left(u_{0}, u_{1}\right) \leq C(\Omega) \mu\|\nabla u(t)\|_{L^{2}(\Omega)}^{4}+4 E\left(u_{0}, u_{1}\right)$, so $0<-4 E\left(u_{0}, u_{1}\right)(C(\Omega) \mu-$ $\beta)^{-1} \leq\|\nabla u(t)\|_{L^{2}(\Omega)}^{4}$ holds as long as the strong solution $u(t)$ exists. Moreover, if $v \in H^{2}(\Omega) \cap H_{0}^{1}(\Omega)$ satisfies $J(v)=(\beta / 4)\|\nabla v\|_{L^{2}(\Omega)}^{4}-(\mu / 4)\|v\|_{L^{4}(\Omega)}^{4}<$ 0 , then for any $\varepsilon>0$ we have $E(\varepsilon v, 0)=J(\varepsilon v)=\varepsilon^{4} J(v)<0$, so the strong solution of (1.3)-(1.4) and (1.2) with $\left(u_{0}, u_{1}\right)=(\varepsilon v, 0)$ blows up in a finite time by Theorem 3.3.

Finally, we note that our abstract result is also applicable to the problem for $\Omega=\mathbf{R}^{n}$ :

$$
u_{t t}+\delta u_{t}-\Delta u+m u-|u|^{q-2} u=0, \quad t \geq 0, \quad x \in \mathbf{R}^{n}
$$

where $m>0$ and $q>2$. From Theorem 1.2, we have the following result.

Theorem 3.4 Let $\delta>0, m>0, q>2$ and $q<2 n /(n-2)$ if $n \geq 3$. If $\left(u_{0}, u_{1}\right) \in H^{2}\left(\mathbf{R}^{n}\right) \times H^{1}\left(\mathbf{R}^{n}\right)$ satisfies $E\left(u_{0}, u_{1}\right)<d$ and $K\left(u_{0}\right)<0$, then the strong solution of (3.4)-(1.2) can not be continued to $[0,+\infty)$ as a strong solution of (3.4)-(1.2).

Theorem 3.4 gives the instability of ground states (least energy stationary solutions) of (3.4) as well as the case of $\delta=0$ by Berestycki and Cazenave [2] (see also Keller [10] for a related result).

\section{References}

[1] Ball J.M., Remarks on blow-up and nonexistence theorems for nonlinear evolution equations. Quart. J. Math. Oxford 28 (1977), 473-486.

[2] Berestycki H. and Cazenave T., Instabilité des états stationnaires dans les équations de Schrödinger et de Klein-Gordon non linéaires. C. R. Acad. Sci. 
Paris 293 (1981), 489-492.

[3] Browder F.E., On non-linear wave equations. Math. Z. 80 (1962), 249-264.

[4] Fujii A. and Ohta M., Asymptotic behavior of blowup solutions of a parabolic equation with the p-Laplacian. Publ. RIMS, Kyoto Univ. (to appear).

[5] Glassey R.T., Blow-up theorems for nonlinear wave equations. Math. Z. 132 (1973), 183-203.

[6] Ikehata R., A note on the global solvability of solutions to some nonlinear wave equations with dissipative terms. Differential and Integral Equations 8 (1995), 607-616.

[7] Ikehata R. and Okazawa N., A class of second order quasilinear evolution equations. J. Differential Equations 114 (1994), 106-131.

[8] Ikehata R. and Suzuki T., Stable and unstable sets for evolution equations of parabolic and hyperbolic type. Hiroshima Math. J. (to appear).

[9] Ishii H., Asymptotic stability and blowing up of solutions of some nonlinear equations. J. Differential Equations 26 (1977), 291-319.

[10] Keller C., Large-time asymptotic behavior of solutions of nonlinear wave equations perturbed from a stationary ground state. Comm. Partial Differential Equations 8 (1983), 1073-1099.

[11] Levine H.A., Instability and nonexistence of global solutions to nonlinear wave equations of the form $P u_{t t}=-A u+F(u)$. Trans. Amer. Math. Soc. 192 (1974), $1-21$.

[12] Li T.-T. and Zhou Y., Breakdown of solutions to $\square u+u_{t}=|u|^{1+\alpha}$. Preprint.

[13] Mizoguchi N., Ninomiya H. and Yanagida E., Diffusion-induced blowup in a nonlinear parabolic system. Preprint.

[14] Payne L.E. and Sattinger D.H., Saddle points and instability of nonlinear hyperbolic equations. Israel J. Math. 22 (1975), 273-303.

[15] Souplet Ph., Nonexistence of global solutions to some differential inequalities of the second order and applications. Portugaliae Math. 52 (1995), 289-299.

[16] Tsutsumi M., On solutions of semilinear differential equations in a Hilbert space. Math. Japon. 17 (1972), 173-193.

[17] Zhou Y., Blow up of classical solutions to $\square u=|u|^{1+\alpha}$ in three space dimensions. J. Partial Differential Equations 5 (1992) 21-32.

Graduate School of Mathematical Sciences

The University of Tokyo

Komaba, Tokyo 153, Japan

E-mail: ohta@ms326yzk.ms.u-tokyo.ac.jp 